\title{
FIELDS OF VALUES OF A MATRIX
}

\author{
WALLACE GIVENS
}

A complex number $\rho$ is said to belong to the field of values $F_{I}(A)$ of a square matrix $A$ with real or complex elements if

$$
\rho_{I}=\frac{(x, A x)_{I}}{(x, x)_{I}}
$$

for some vector $x$, where $(x, x)_{I} \equiv \bar{x}_{1} x_{1}+\cdots+\bar{x}_{n} x_{n}$. It is known that $F_{I}(A)$ is a closed bounded convex region containing the characteristic values $\lambda_{1}, \cdots, \lambda_{n}$ of $A$ and with these points as foci of the algebraic curve bounding $F_{I}(A)$. A summary of results and a bibliography has recently been given by Parker [1] ${ }^{1}$ who observes that several authors have given bounds for the roots which are actually bounds for $F_{I}(A)$.

Since $F_{I}(A)$ is invariant when $A$ is replaced by $B=U A U^{-1}$ for $U$ unitary but may be altered if $B=T A T^{-1}$ for arbitrary nonsingular $T$, information about $F_{I}(A)$ cannot be expected to give precise information on the $\lambda_{i}$. Evidently the difficulty is that $F_{I}(A)$ depends not only on the linear mapping described by $A$ but also involves a choice of metric which is unrelated to the linear mapping. If, however, we define for each positive definite hermitian matrix $H$, a field of values $F_{H}(A)$ consisting of all complex numbers

$$
\rho_{H}=\frac{x^{*} H A x}{x^{*} H x} \equiv \frac{(x, A x)_{H}}{(x, x)_{H}}
$$

obtainable from a column vector $x$ and its conjugate transposed (row) vector $x^{*}$, we have a class of regions in the Argand plane depending only on $A$. The main purpose of this note is to show that the intersection of all the $F_{H}(A)$ is the minimum convex polygon $P(A)$ containing all the roots of $A$. This opens the possibility that by a judicious choice of $H$, one may use known results on fields of values to obtain reasonably precise information on at least those $\lambda_{i}$ at the vertices or on the edges of $P(A)$.

1. Setting

$$
H=T^{*} T,
$$

Presented to the Society, November 24, 1951; received by the editors June 20, 1951 and, in revised form, August 2, 1951.

1 Numbers in brackets refer to the bibliography at the end of the paper. 
for $T$ nonsingular, as we can do if and only if $H$ is positive definite hermitian,

$$
\rho_{H}=\frac{(y, B y)_{I}}{(y, y)_{I}},
$$

where $y=T x$ and $B=T A T^{-1}$. Hence, $F_{H}(A) \subseteq F_{I}(B)$ and, taking $x=T^{-1} y$ to get the reverse inclusion, we have the following lemma.

LEMma 1. For any positive definite hermitian matrix $H, F_{H}(A)$ $=F_{I}(B)$ for $a B$ similar to $A$. Conversely, if $B=T A T^{-1}, F_{I}(B)$ $=F_{H}(A)$ for $H=T^{*} T$.

The field of values of a fixed matrix relative to the various metrics are therefore the same as the fields of values of all matrices similar to the fixed one when they are taken with respect to a single metric. Explicitly, if $A$ and $B$ are any two similar matrices and $H$ and $K$ are any two positive definite hermitian matrices, there exists a matrix $C$ similar to $A$ and $B$ and a positive definite hermitian matrix $L$ such that

$$
F_{K}(B)=F_{H}(C)=F_{L}(A) .
$$

2. Every $F_{H}(A)$ contains all the roots $\lambda_{i}$ of $A$ so that $F_{H}(A) \supseteq P(A)$ for every $H$. Toeplitz [2], in his paper initiating the subject, observed that when $A$ is normal (so that it is unitarily similar to a diagonal matrix), $F_{I}(A)=P(A)$. It is natural to say that $A$ is normal with respect to $H=T^{*} T$ if $T A T^{-1}$ is normal in the usual sense. This definition is independent of the factorization of $H$ since if $H=V^{*} V$, it follows that $V A V^{-1}=U\left(T A T^{-1}\right) U^{-1}$ for $U=V T^{-1}$ unitary and so $V A V^{-1}$ is normal if $T A T^{-1}$ is. Indeed, $A$ is normal with respect to $H$ if $(H A)\left(A H^{-1}\right)^{*}=(H A)^{*}\left(A H^{-1}\right)$. For $A$ to be normal with respect to some $H$, it is necessary and sufficient that all its elementary divisors be simple and then $F_{H}(A)=P(A)$. This sufficient condition for $F_{H}(A)=P(A)$ is not necessary, the precise result being given in Theorem 2 below.

We now prove the following lemma.

LEMMA 2. If $A$ has a nonsimple elementary divisor with root $\lambda$, then $\lambda$ is an interior point of $F_{H}(A)$ for every $H$.

Proof. Since the property of having a nonsimple elementary divisor is invariant under similarity and $F_{H}(A)=F_{I}\left(T A T^{-1}\right)$ for $H=T^{*} T$, it is sufficient to show that $F_{I}(A)$ has $\lambda$ as an interior point under the stated hypothesis on $A$. Remembering the Jordan normal form for $A$, there exist linearly independent vectors $e_{1}$ and $e_{2}$ such 
that $A e_{1}=\lambda e_{1}$ and $A e_{2}=\lambda e_{2}+e_{1}$. Choosing unit vectors $f_{1}=\alpha e_{1}$ and $f_{2}$ orthogonal to $f_{1}$ and in the plane of $e_{1}$ and $e_{2}$, we have $A f_{1}=\lambda f_{1}$ and $A f_{2}=\lambda f_{2}+\mu f_{1}$. The requirement that $f_{2}$ be orthogonal to $f_{1}$ prevents us from taking $\mu=1$, but $\mu \neq 0$ and multiplying $f_{1}$ by a number of absolute value one permits requiring $\mu$ real and positive. Then for $x=x_{1} f_{1}+x_{2} f_{2}, A x=\lambda x+\mu x_{2} f_{1}$ and (1) gives

$$
\rho_{I}=\lambda+\frac{\mu \bar{x}_{1} x_{2}}{\bar{x}_{1} x_{1}+\bar{x}_{2} x_{2}}=\lambda+\mu \frac{d e^{i \delta}}{1+d^{2}},
$$

where $x_{2} x_{1}^{-1}=d e^{i \delta}$, or $\rho_{I}=\lambda$ if $x_{1}=0$. Hence these values of $\rho_{I}$ (certainly contained in $F_{I}(A)$ ) are the points on the interior and boundary of a circle with center $\lambda$ and nonzero radius $\mu / 2$. (In case $A$ is two by two with distinct roots, Toeplitz (loc. cit.) observed that $F_{I}(A)$ is an ellipse with the roots as foci.)

The field of values of a direct sum of matrices clearly must contain the fields of values of the components. When the spaces in which the components operate are orthogonal with respect to the given metric, we can state a stronger result.

Lemma 3. Let $A=A_{1} \oplus A_{2} \oplus \cdots \oplus A_{k}$ and $H=H_{1} \oplus H_{2} \oplus \cdots \oplus H_{k}$, where $H_{\alpha}$ and $A_{\alpha}$ are of the same order $(\alpha=1, \cdots, k)$. Then $F_{H}(A)$ is the minimum convex set containing all the $F_{\alpha}\left(A_{\alpha}\right)$.

Proof. Writing $x=x_{1}+x_{2}+\cdots+x_{k}$, where $x_{\alpha}$ has nonzero components only in the rows associated with $A_{\alpha}$ and $H_{\alpha}$,

$$
\rho=\frac{(x, A x)_{H}}{(x, x)_{H}}=\frac{\sum_{\alpha}\left(x_{\alpha}, A_{\alpha} x_{\alpha}\right)_{\alpha}}{\sum_{\beta}\left(x_{\beta}, x_{\beta}\right)_{\beta}}=\frac{\sum_{\alpha} \rho_{\alpha} m_{\alpha}}{\sum_{\alpha} m_{\alpha}},
$$

where

$$
\rho_{\alpha}=\frac{\left(x_{\alpha}, A_{\alpha} x_{\alpha}\right)_{\alpha}}{\left(x_{\alpha}, x_{\alpha}\right)_{\alpha}}
$$

is in $F_{\alpha}\left(A_{\alpha}\right)$,

$$
m_{\alpha}=\frac{\left(x_{\alpha}, x_{\alpha}\right)_{\alpha}}{\sum_{\beta}\left(x_{\beta}, x_{\beta}\right)_{\beta}},
$$

the summation is always taken only over those terms involving $x_{\alpha}$ $\neq 0$ and $F_{\alpha}$ and ()$_{\alpha}$ refer to the subspace with metric $H_{\alpha}$. That is, $\rho$ is the center of mass of a system of non-negative masses $m_{\alpha}$ at the points $\rho_{\alpha}$. Hence, every point of $F_{H}(A)$ is in every convex region 
containing all $F_{\alpha}\left(A_{\alpha}\right)$ and the lemma follows.

3. We can now prove the following theorem.

THEOREM 1. The intersection of the regions $F_{H}(A)$ for all positive definite $H$ is the minimum convex polygon, $P(A)$, containing all the roots of $A$.

Proof. We first suppose that $A$ has only a single root, say $\lambda$, and hence is of the form $A=\lambda 1_{m}+N$, where $N$ is nilpotent. Since

$$
\frac{(x, A x)_{H}}{(x, x)_{H}}=\lambda+\frac{(x, N x)_{H}}{(x, x)_{H}},
$$

it will suffice to show that for a suitable choice of $H, F_{H}(N)$ is contained in a circle of arbitrarily small radius $\epsilon$ about the origin. Since $\delta N$ is similar to $N$ for any nonzero $\delta$, there exists a $T$ such that $T N T^{-1}$ $=\delta N$, where we choose $\delta$ so that

$$
\delta \cdot\left[\text { Maximum of }|\tau| \text { for all } \tau \in F_{I}(N)\right]<\epsilon .
$$

Then, by Lemma $1,|\sigma|<\epsilon$ for every $\sigma \in F_{H}(N)$, where $H=T^{*} T$.

Writing an arbitrary matrix $A$ as a direct sum of matrices each of which has only a single root, and using Lemma $3, F_{H}(A)$ is the minimum convex region containing regions about the $\lambda_{i}$ which can be made (independently) arbitrarily small. Hence we have Theorem 1.

TheOREM 2. A necessary and sufficient condition that $F_{H}(A)=P(A)$ for some positive definite hermitian matrix $H$ is that the elementary divisors corresponding to roots lying on the boundary of $P(A)$ shall all be simple.

Lemma 2 shows the condition to be necessary. To prove sufficiency we can replace $A$ by a similar matrix of the form $A=A_{1} \oplus A_{2}$, where $A_{1}$ is diagonal with roots on the boundary of $P(A)$ and $A_{2}$ has all its roots in the interior of $P(A)$. Taking $H=H_{1} \oplus H_{2}, F_{1}\left(A_{1}\right)$ is $P(A)$ for $H_{1}$ a unit matrix and, by the argument of Theorem $1, H_{2}$ can be chosen so that $F_{2}\left(A_{2}\right)$ is interior to $P(A)$.

\section{BIBLIOGRAPHY}

1. W. V. Parker, Characteristic roots and fields of values of a matrix, Bull. Amer. Math. Soc. vol. 57 (1951) pp. 103-108.

2. O. Toeplitz, Das algebraische Analogon zu einem Satz von Fejer, Math. Zeit. vol. 2 (1918) pp. 187-197.

OAK Ridge National Laboratory and UNIVERSITY OF TENNESSEE 